\title{
CoxIter - Computing invariants of hyperbolic Coxeter groups
}

\author{
R. Guglielmetti
}

\begin{abstract}
CoxIter is a computer program designed to compute invariants of hyperbolic Coxeter groups. Given such a group, the program determines whether it is cocompact or of finite covolume, whether it is arithmetic in the non-cocompact case, and whether it provides the Euler characteristic and the combinatorial structure of the associated fundamental polyhedron. The aim of this paper is to present the theoretical background for the program. The source code is available online as supplementary material with the published article and on the author's website (http://coxiter.rgug.ch).

Supplementary materials are available with this article.
\end{abstract}

\section{Introduction}

Let $\mathcal{H}^{n}$ be the hyperbolic $n$-space, and let Isom $\mathcal{H}^{n}$ be the group of isometries of $\mathcal{H}^{n}$. For a given discrete hyperbolic Coxeter group $\Gamma<\operatorname{Isom} \mathcal{H}^{n}$ and its associated fundamental polyhedron $P \subset \mathcal{H}^{n}$, we are interested in geometrical and combinatorial properties of $P$. We want to know whether $P$ is compact, has finite volume and, if the answer is yes, what its volume is. We also want to find the combinatorial structure of $P$, namely, the number of vertices, edges, 2-faces, and so on. Finally, it is interesting to find out whether $\Gamma$ is arithmetic, that is, if $\Gamma$ is commensurable to the reflection group of the automorphism group of a quadratic form of signature $(n, 1)$. Most of these questions can be answered by studying finite and affine subgroups of $\Gamma$, but this involves a huge number of computations.

This article presents the algorithms used in CoxIter, a computer program written in $\mathrm{C}++$ designed to compute these invariants. The program is published under a free license (the GNU General Public License v3) and can be used freely in various projects. The source code and the documentation are available as supplementary material with the online version of this article and on the author's website.

The input of CoxIter is the graph of a hyperbolic Coxeter group (encoded in a simple way in a text file, see Appendix A) and a typical output can be the following.

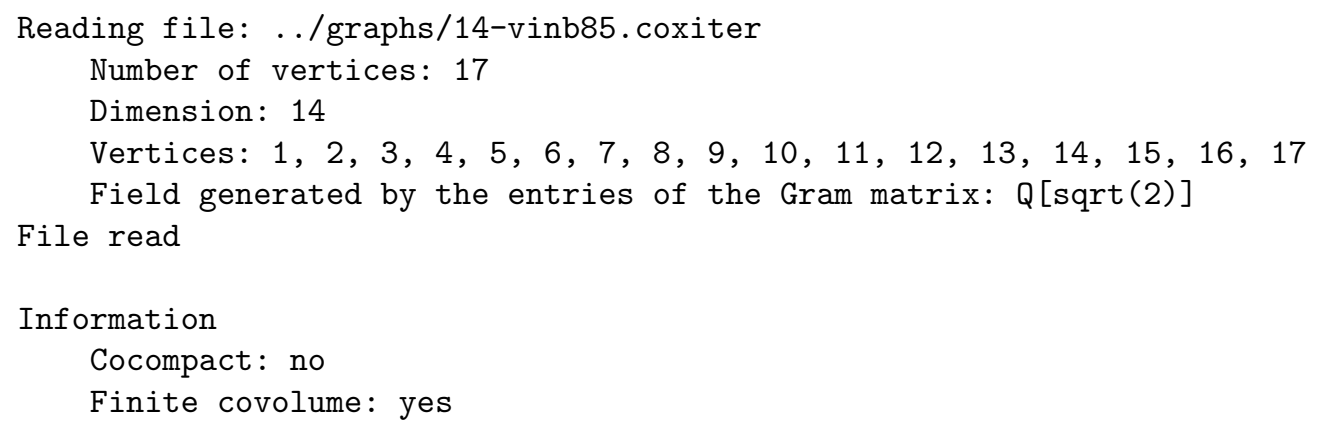

Received 5 January 2015; revised 28 July 2015.

2010 Mathematics Subject Classification 5104 (primary), 52B05, $20 \mathrm{~F} 55$ (secondary).

Supported by the Schweizerischer Nationalfonds SNF no. 200020_144438/1. 


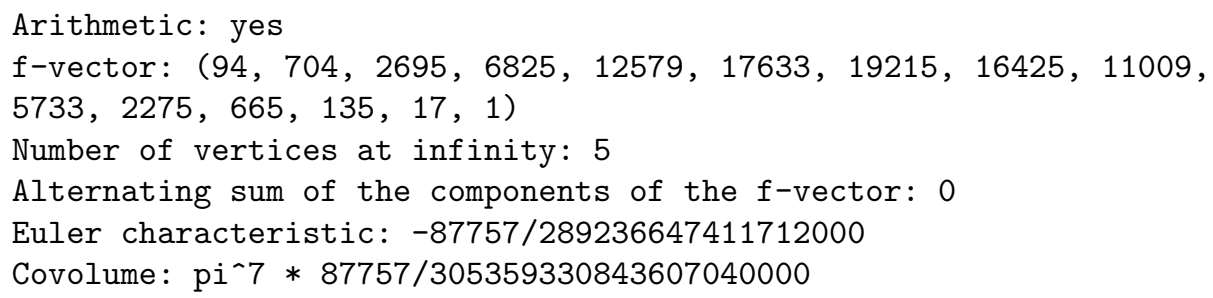

In the first section, we give the theoretical background that is needed to present the algorithms and their implementation in the program. These algorithms are explained in the second section. In the third part, we give some applications of CoxIter: we use the program to compute the invariants of a few Coxeter graphs in higher dimensions, including recently found groups of Vinberg in dimension eighteen. In the last section, we explain how CoxIter was tested.

\section{Theoretical background}

In this section we present the theoretical tools needed to implement the algorithms in CoxIter.

\subsection{Hyperbolic space}

We denote by $\mathbb{R}^{n, 1}$ the space $\mathbb{R}^{n+1}$ endowed with the Lorentzian product given by

$$
\left(x=\left(x_{0}, x_{1}, \ldots, x_{n}\right), y=\left(y_{0}, y_{1}, \ldots, y_{n}\right)\right) \longmapsto\langle x, y\rangle=-x_{0} y_{0}+\sum_{i=1}^{n} x_{i} y_{i} .
$$

For a vector $x \in \mathbb{R}^{n, 1}$, we call the scalar $\|x\|^{2}:=\langle x, x\rangle \in \mathbb{R}$ the square of the Lorentzian norm of $x$. Then, we let $\mathcal{H}^{n} \subset \mathbb{R}^{n, 1}$ be the set defined by

$$
\mathcal{H}^{n}:=\left\{x \in \mathbb{R}^{n, 1}:\langle x, x\rangle=-1, x_{0}>0\right\} \subset \mathbb{R}^{n, 1} .
$$

If we endow $\mathcal{H}^{n}$ with the map $d: \mathcal{H}^{n} \times \mathcal{H}^{n} \longrightarrow \mathbb{R}$, which sends $(x, y)$ to $\operatorname{arcosh}(-\langle x, y\rangle)$, then $\left(\mathcal{H}^{n}, d\right)$ is a metric space, which is the Minkowski model of the hyperbolic $n$-space. The boundary of $\mathcal{H}^{n}$ can be identified with the set

$$
\partial \mathcal{H}^{n}=\left\{x \in \mathbb{R}^{n, 1}:\langle x, x\rangle=0, \sum_{i=0}^{n} x_{i}^{2}=1, x_{0}>0\right\},
$$

and we let $\overline{\mathcal{H}^{n}}:=\partial \mathcal{H}^{n} \cup \mathcal{H}^{n}$.

A hyperplane of $\mathcal{H}^{n}$ is given by the intersection of the orthogonal complement (with respect to the Lorentzian product) of a vector $v$ of Lorentzian norm one and $\mathcal{H}^{n}$. We denote such a hyperplane by $H_{v}$. Note that such a hyperplane splits $\mathcal{H}^{n}$ into two half-spaces $H_{v}^{+}:=\{x \in$ $\left.\mathcal{H}^{n}:\langle v, x\rangle \geqslant 0\right\}$ and $H_{v}^{-}:=\left\{x \in \mathcal{H}^{n}:\langle v, x\rangle \leqslant 0\right\}$, whose intersection is $H_{v}$. The relative behaviour of two distinct hyperplanes $H_{v}$ and $H_{w}$ can be described by means of the Lorentzian product of $v$ and $w$ (see [17, Theorems 3.2.6, 3.2.7 and 3.2.9] or [23, $\S 1.1]$ ).

- The hyperplanes intersect if and only if $|\langle v, w\rangle|<1$. In this case, the acute dihedral angle between them is given by $\arccos (|\langle v, w\rangle|)$.

- The hyperplanes are parallel if and only if $|\langle v, w\rangle|=1$. In this case their dihedral angle is zero.

- The hyperplanes are ultraparallel if and only if $|\langle v, w\rangle|>1$. In this setting, the two hyperplanes share a common perpendicular of length $\operatorname{arcosh}(\mid\langle v, w \mid\rangle)$. 


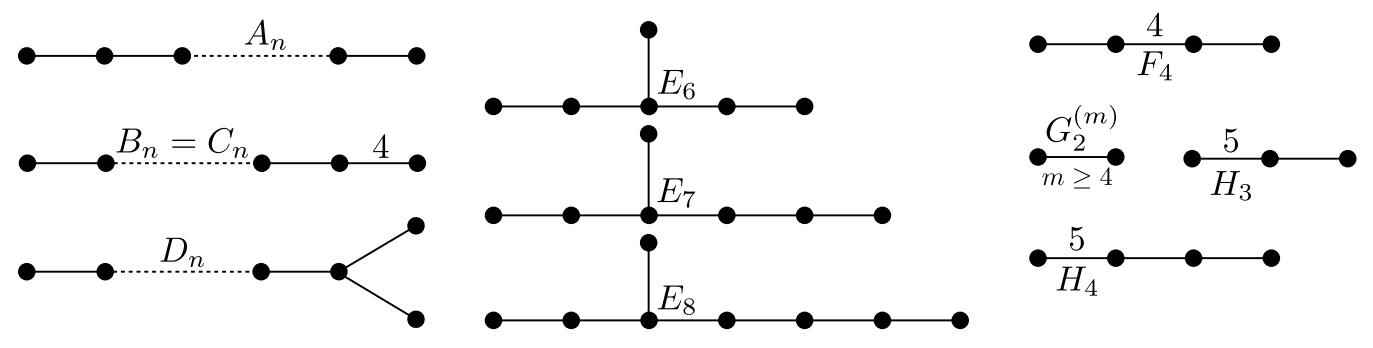

Figure 1. Irreducible finite Coxeter groups.

\subsection{Abstract Coxeter groups}

Definition 1. A Coxeter group is a finitely presented group given by the presentation

$$
\left\langle s_{1}, \ldots, s_{d}:\left(s_{i} s_{j}\right)^{m_{i j}}=1\right\rangle,
$$

where $m_{i j}=1$ if and only if $i=j$, and $m_{i j}=m_{j i} \in\{2,3, \ldots\} \cup\{\infty\}$ if $i \neq j$. By $m_{i j}=\infty$, we mean that there is no relation between $s_{i}$ and $s_{j}$. If $\Gamma$ is such a group and $S:=\left\{s_{1}, \ldots, s_{d}\right\}$ is the set of generators, we will refer to the group as $(\Gamma, S)$, or just $\Gamma$ if there is no ambiguity on $S$. When it is convenient, we use the notation $m\left(s_{i}, s_{j}\right):=m_{i j}$.

If $S^{\prime}$ is a subset of $S$, we denote the subgroup of $\Gamma$ generated by the elements of $S^{\prime}$ by $\Gamma_{S^{\prime}}$.

Definition 2. An easy way to represent a Coxeter group $\left(\Gamma, S=\left\{s_{1}, \ldots, s_{d}\right\}\right)$ is by its Coxeter diagram, or Coxeter graph. The Coxeter diagram of $\Gamma$ is the graph whose vertices correspond to the elements of $S$. Moreover, there is an edge between the vertices $s_{i}$ and $s_{j}$ if and only if $m\left(s_{i}, s_{j}\right) \geqslant 3$. We label such an edge with $m\left(s_{i}, s_{j}\right)$ if $m\left(s_{i}, s_{j}\right) \geqslant 4$. Sometimes, instead of labelling the edge, we use a double edge if $m\left(s_{i}, s_{j}\right)=4$ and a bold (or heavy) edge if $m\left(s_{i}, s_{j}\right)=\infty$.

Notation 1. Let $\left(\Gamma, S=\left\{s_{1}, \ldots, s_{d}\right\}\right)$ be a Coxeter group. We can associate to $(\Gamma, S)$ the real $d \times d$ matrix $G(\Gamma, S)=\left(g_{i j}\right) \in \operatorname{Mat}(d, \mathbb{R})$ defined as follows: $g_{i i}=1, g_{i j}=-1$ if $m\left(s_{i}, s_{j}\right)=\infty$ and $g_{i j}=-\cos \left(\pi / m\left(s_{i}, s_{j}\right)\right)$ otherwise.

\section{EXAMPLES 1.}

- It is well known that the symmetric group $S_{n}$ is isomorphic to the Coxeter group $A_{n-1}$ (see Figure 1): the $i$ th node of $A_{n-1}$ corresponds to the transposition $(i, i+1)$ of $S_{n}$.

- The dihedral group of $2 n$ elements is isomorphic to $G_{2}^{(n)}$ (see Figure 1).

- If $P$ is a polyhedron (see Definition 4) in $\mathbb{R}^{n}$ whose dihedral angles are integer submultiples of $\pi$ and if $\Gamma<$ Isom $\mathbb{R}^{n}$ is the (discrete) group generated by the reflections in the facets of $P$, then $\Gamma$ is a Coxeter group.

Definition 3. A Coxeter group is said to be irreducible if its Coxeter diagram is connected.

It is easy to see that a Coxeter group is the product of the groups corresponding to the connected components of its Coxeter diagram.

The finite irreducible Coxeter groups were classified by Coxeter [4] by means of their Coxeter diagrams. The classification is presented in Figure 1.

\subsection{Reflection groups}

In this section, we present the connection between abstract Coxeter groups and discrete reflection groups in the facets (sides of codimension one) of convex finite-sided polyhedra. 
In what follows, $X$ denotes the unit $n$-sphere $S^{n}$, the Euclidean $n$-space $E^{n}$ or $\mathcal{H}^{n}$.

A vector $v \in E^{n}$ and a real number $t \in \mathbb{R}$ determine an (affine) hyperplane $H_{v, t}:=\{x \in$ $\left.E^{n}:\langle x, v\rangle=t\right\}$, denoted by $H_{v}$ if $t=0$, and such a hyperplane splits $E^{n}$ into two half-spaces $H_{v, t}^{+}:=\left\{x \in E^{n}:\langle v, x\rangle \geqslant t\right\}$ and $H_{v, t}^{-}:=\left\{x \in E^{n}:\langle v, x\rangle \leqslant t\right\}$, whose intersection is $H_{v, t}$. A hyperplane in $S^{n}$ is the intersection of a hyperplane $H_{v}$ of $E^{n+1}$ with $S^{n}$.

Definition 4. A polyhedron $P$ in $X$ is a subset of $X$ which is the intersection of a finite number of half-spaces, each one bounded by a hyperplane. Moreover, we require that $P$ has non-empty interior and that no half-space contains the intersection of the others.

Definition 5. Let $H_{v}$ be a hyperplane of $X$. The reflection $r_{v}=r_{H_{v}}$ associated to the hyperplane $H_{v}$ is defined as (recall that we choose $v$ with norm one)

$$
\begin{aligned}
r_{H_{v}}: X & \longrightarrow X \\
x & \longmapsto x-2\langle x, v\rangle v .
\end{aligned}
$$

Definition 6. Let $P=\bigcap_{i=1}^{d} H_{v_{i}}^{-}$be a polyhedron in $X$ and $\Gamma \leqslant \operatorname{Isom} X$. We say that $\Gamma$ is the reflection group associated to $P$ if $\Gamma$ is generated by the reflections in the facets of $P$ : that is, $\Gamma=\left\langle r_{H_{v_{1}}}, \ldots, r_{H_{v_{d}}}\right\rangle$.

Let $P \subset X$ be a polyhedron and let $\Gamma \leqslant$ Isom $X$ be its associated reflection group. If $\Gamma$ is discrete, then all the dihedral angles of $P$ are submultiples of $\pi$ (that is, zero or of the form $\pi / k$ where $k \in \mathbb{Z}$ ). For any facet $S_{i}$ of $P$, we let $H_{v_{i}}$ be the hyperplane containing $S_{i}$ such that $P \subset H_{v_{i}}^{-}$. Now, if the dihedral angle between two adjacent facets $S_{i}$ and $S_{j}$ of $P$ is $\pi / \theta\left(S_{i}, S_{j}\right)$, then the order of $r_{v_{i}} \circ r_{v_{j}}$ in $\Gamma$ is $\theta\left(S_{i}, S_{j}\right)$ (see [17, Theorem 7.1.2]). Suppose, now, that $P$ is of finite volume and has facets $\mathcal{S}=\left\{S_{1}, \ldots, S_{d}\right\}$. Then, by [17, Theorem 7.1.4],

$$
\left\langle r_{1}, \ldots, r_{d} \mid r_{i}^{2}=1,\left(r_{i} \cdot r_{j}\right)^{\theta\left(S_{i}, S_{j}\right)}=1\right\rangle \cong \Gamma .
$$

The matrix $G=G(\Gamma, S)$ of a Coxeter group $(\Gamma, S)$ (see Notation 1) induces a quadratic form called the Tits form on $\mathbb{R}^{d}$, where $d=|S|$, via $x \longmapsto x^{t} G x$. If the matrix $G$ is indecomposable (meaning that we cannot transform $G$ to a block diagonal matrix with permutations of the rows and columns of $G$ ) or, equivalently, if the graph of the group is connected, then we get information about the group by looking at the signature $(n, p, q)$ of the quadratic form, where $n$ (respectively, $p, q$ ) is the number of positive (respectively, negative, zero) eigenvalues of $G$. For a positive $n$, we are interested in the following cases.

$p=q=0$ (the quadratic form is positive definite). In this case, the group is finite (see the classification of irreducible finite Coxeter groups in Figure 1). It can be shown that $\Gamma$ can be realized as a discrete group of isometries of the $n$-sphere $S^{n}$. Thus, $\Gamma$ is called spherical.

$p=0, q>0$ (the quadratic form is positive semidefinite). The group can be realized as a discrete subgroup of Isom $E^{n}$. Hence, it is said to be affine (or euclidean or parabolic). The classification of irreducible affine Coxeter groups is given in Figure 2 (see, for example, [2, Chapter $6, \S 4.3$, Theorem 4]).

$p=1, q \geqslant 0$. The group can be realized as a discrete subgroup of Isom $\mathcal{H}^{n}$ (see details in $\S 1.4$ ). Thus, it is called hyperbolic.

Now, if the matrix $G$ is decomposable into a diagonal block matrix with blocks $G_{1}, \ldots, G_{l}$, we say that the group is spherical (respectively, affine) if each block $G_{i}$ is spherical (respectively, 


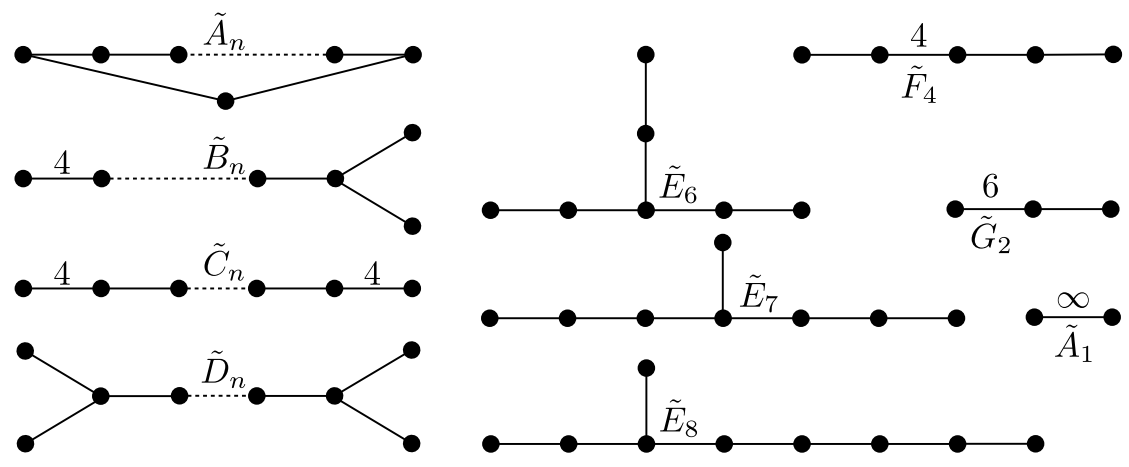

Figure 2. Irreducible affine Coxeter groups.

affine). If the matrix $G$ has signature $(n, 1, q)$ for some $n$ and $q$, we say that the group is hyperbolic.

If $\mathcal{G}$ is the Coxeter diagram of $\Gamma$, then a subdiagram of $\mathcal{G}$ is called spherical (respectively, affine, hyperbolic) if the corresponding subgroup of $\Gamma$ is spherical (respectively, affine, hyperbolic).

\subsection{Hyperbolic Coxeter groups and hyperbolic Coxeter polyhedra}

In this section, we present some concepts related to hyperbolic Coxeter groups and polyhedra. Let $P \subset \mathcal{H}^{n}$ be a polyhedron. Hence, there exist vectors $v_{1}, \ldots, v_{r}$ of Lorentzian norm one and hyperplanes $H_{i}=\left\langle v_{i}\right\rangle^{\perp}$ such that $P=\bigcap_{i=1}^{r} H_{i}^{-}$, where $H_{i}^{-}$is the half-space delimited by $H_{i}$ and given by $H_{i}^{-}:=\left\{x \in \mathcal{H}^{n}:\left\langle x, v_{i}\right\rangle \leqslant 0\right\}$.

REMARK 1. Unlike in the Euclidean space, there exist unbounded hyperbolic Coxeter polyhedra of finite volume. Such a polyhedron is the convex hull of a finite set of points $x_{1}, \ldots, x_{k} \in \overline{\mathcal{H}^{n}}$ with at least one $x_{i} \in \partial \mathcal{H}^{n}$. Such a vertex is called a vertex at infinity of the polyhedron (see $[\mathbf{1 7}, \S 6.4]$ for more details).

Definition 7. The Gram matrix of the polyhedron $P \subset \mathcal{H}^{n}$ is the matrix $G=G(P) \in$ $\operatorname{Mat}(r, \mathbb{R})$ defined as $G=\left(\left\langle v_{i}, v_{j}\right\rangle\right)_{1 \leqslant i, j \leqslant r}$.

Definition 8. Suppose now that $P$ is a hyperbolic Coxeter polyhedron, that is, the dihedral angles are either zero or submultiples of $\pi$. The Coxeter diagram, or Coxeter graph, of $P$ is the graph whose vertices $s_{i}$ correspond to the hyperplanes $H_{i}$. For two hyperplanes $H_{i}$ and $H_{j}$, there is between the vertices $s_{i}$ and $s_{j}$ :

- a dotted line if $H_{i}$ and $H_{j}$ are ultraparallel (labelled by the hyperbolic cosine of the length of the common perpendicular of the two hyperplanes);

- a line labelled with $\infty$ if $H_{i}$ and $H_{j}$ are parallel (we use sometimes a bold, or heavy, edge with no labelling); and

- a line if the dihedral angle is $\pi / m_{i j}$ with $m_{i j} \geqslant 3$, which is simple if $m_{i j}=3$ and is labelled by $m_{i j}$ if $m_{i j}>3$. Note that, sometimes, instead of labelling the edge, we use a double edge if $m_{i j}=4$.

Note that we do not distinguish between the Gram matrix of a hyperbolic Coxeter polyhedron, the corresponding Coxeter diagram and the associated hyperbolic Coxeter group.

There is the following natural question: given a Coxeter graph, is it possible to find a hyperbolic Coxeter polyhedron whose graph is the given one? The answer is yes if the Gram 
matrix of the graph has the correct signature, as explained in the next theorem.

Theorem $1.1\left[\mathbf{2 3}\right.$, Theorem 2.1]. Let $G=\left(G_{i j}\right)$ be an indecomposable symmetric matrix of signature $(n, 1, k)$ such that:

$-G_{i i}=1$ for every $i$; and

$-G_{i j} \leqslant 0$ for every $i \neq j$.

Then, there exists a convex polyhedron $P$ in $\mathcal{H}^{n}$ whose Gram matrix is equal to G. Moreover, $P$ is unique up to isometry. We will refer to $P$ as the polyhedron associated to $G$.

Therefore, given a hyperbolic Coxeter group $\Gamma$, we can speak of geometrical properties of its associated polyhedron $P \subset \mathcal{H}^{n}$. If the polyhedron $P$ is compact, then the group $\Gamma$ is called cocompact. Note that there is a criterion to decide if $\Gamma$ is cocompact or not (see $\S 1.6)$. In a similar way, if $P$ is of finite volume (with respect to the hyperbolic metric), we say that $\Gamma$ is cofinite or of finite covolume. In this case, the covolume of $\Gamma$ is the volume of $P$. As before, there is a nice criterion to decide whether the group is of finite covolume or not (see $\S 1.6$ ).

It has been shown that hyperbolic Coxeter groups of finite covolume do not exist in dimensions above 995 (see [16, Theorem C]), but examples of such groups are known only up to dimension 21 (see [1, Example 5]). In the cocompact case, it is known that such groups do not exist if the dimension is greater than 29 (see [22, Theorem 1]). However, examples of such groups are known only up to dimension 8 (see [3] for the arithmetic cocompact hyperbolic Coxeter group in dimension 8 and [14] for a list of cocompact groups in dimensions $5-8$ ).

\subsection{Euler characteristic, $f$-vector and volume}

In what follows, unless stated otherwise, $\Gamma<$ Isom $\mathcal{H}^{n}$ denotes a hyperbolic Coxeter group with finite set of generator $S$. Let $P$ be its associated fundamental convex polyhedron.

Notation 2. Let $G$ be a finitely generated group with generating set $S$. We denote by $l_{S}$ the length function of $G$ with respect to $S$ : for an element $g \in G$,

$$
l_{S}(g)=\min \left\{k \in \mathbb{N}: \exists g_{1}, \ldots, g_{k} \in S \cup S^{-1} \text { such that } g=g_{1} \cdot \ldots \cdot g_{k}\right\} .
$$

Definition 9. Let $G$ be a finitely generated group with generating set $S$. The growth series of $G$ is the formal power series $f_{S}(x)=\sum_{g \in G} x^{l_{S}(g)}$. This series is also called the Poincaré series of $G$.

The next proposition gives the key tool which is used by CoxIter to compute the orbifold Euler characteristic of $\mathcal{H}^{n} / \Gamma$.

Proposition $1.2[\mathbf{1 2},(1.2)$ and (1.3)]. Let $(\Gamma, S)$ be an abstract Coxeter group and let $\mathcal{F}=\left\{T \subset S: \Gamma_{T}\right.$ is finite $\}$. Then

$$
\chi(\Gamma)=\sum_{T \in \mathcal{F}} \frac{(-1)^{|T|}}{f_{T}(1)},
$$

where $f_{T}$ is the growth series of the group $\Gamma_{T} \leqslant \Gamma$ generated by $T$ (see Definition 1).

In order to compute $\chi(\Gamma)$, we see, by using the classification of finite Coxeter groups (see Figure 1), that it is sufficient to know the value $f_{T}(1)$ for all irreducible finite Coxeter groups. With the growth series (see [12, Table 1], for example), we find the values given in Table 1 , page 760 .

The next result relates the Euler characteristic of a hyperbolic Coxeter group $\Gamma<\operatorname{Isom} \mathcal{H}^{n}$ to its covolume when $n$ is even. 
Proposition $1.3[\mathbf{1 2},(1.4)]$. When $n$ is even,

$$
\text { covolume }(\Gamma)=(-1)^{n / 2} \cdot \frac{\pi^{n / 2} \cdot 2^{n} \cdot(n / 2) !}{n !} \cdot \chi(\Gamma) .
$$

REMARK 2. When $n$ is odd, $\chi(\Gamma)=0$.

We are also interested in the combinatorial properties of the Coxeter polyhedron $P$ associated to $\Gamma$. Thus, we consider the vector $\left(f_{0}, \ldots, f_{n-1}, 1\right) \in \mathbb{Z}^{n+1}$, where $f_{i}$ is the number of faces of dimension $i$ of $P$. This vector is called the $f$-vector of $P$ (or $f$-vector of $\Gamma$ ). To compute it, we will use the following results.

Theorem 1.4 [23, Theorem 3.1]. Let $\mathcal{G}$ be the Coxeter diagram of $\Gamma$. There is a bijective correspondence between spherical subdiagrams of rank $k$ of $\mathcal{G}$ and faces of codimension $k$ of $P$.

Theorem 1.5 [23, Theorem 3.2]. Let $\mathcal{G}$ be the Coxeter diagram of $\Gamma$. There is a bijective correspondence between parabolic subdiagrams of rank $n-1$ of $\mathcal{G}$ and vertices at infinity of $P$.

As a test for the output of the program we also use the following classical result of EulerSchläfli.

Proposition 1.6 [15]. For a polyhedron (not necessarily in $\mathcal{H}^{n}$ ), we have the equality

$$
\sum_{i=0}^{n} f_{i}=1-(-1)^{n} .
$$

1.6. Compactness and finite volume criterion

Since a polyhedron $P$ is compact if and only if it is the convex hull of a finite number of vertices in $\mathcal{H}^{n}$ (also called ordinary vertices), we have the following result.

Proposition 1.7 [23, Proposition 4.2]. The polyhedron $P$ is compact if and only if the following conditions are satisfied.

- $P$ contains at least one vertex (that is, face of dimension zero) in $\mathcal{H}^{n}$.

- For every vertex of $P$ and every edge of $P$ emanating from it there is another vertex of $P$ on that edge.

Since a polyhedron $P$ is of finite volume if and only if it is the convex hull of a finite number of vertices in $\overline{\mathcal{H}^{n}}$, we have the following result.

Proposition 1.8 [23, Proposition 4.2]. The polyhedron $P$ has finite volume if and only if the following conditions are satisfied.

TABLE 1. Orders of finite Coxeter groups.

\begin{tabular}{lllr}
\hline Group & $f_{S}(1)$ & Group & \multicolumn{1}{c}{$f_{S}(1)$} \\
\hline$A_{m}$ & $(m+1) !$ & $E_{6}$ & 51840 \\
$B_{m}$ & $2^{m} \cdot m !$ & $E_{7}$ & 2903040 \\
$D_{m}$ & $2^{m-1} \cdot m !$ & $E_{8}$ & 696729600 \\
$G_{m}$ & $2 m$ & $H_{3}$ & 120 \\
$F_{4}$ & 1152 & $H_{4}$ & 14400 \\
\hline
\end{tabular}


- $P$ contains at least one vertex (ordinary or at infinity).

- For every vertex (ordinary or at infinity) of $P$ and every edge of $P$ emanating from it there is another vertex of $P$ (ordinary or at infinity) on that edge.

Using Theorems 1.4 and 1.5 we deduce the following two criteria.

Proposition 1.9 (Cocompactness criterion in CoxIter). Let $\Gamma<\operatorname{Isom} \mathcal{H}^{n}$ be a Coxeter group and $\mathcal{G}$ be its Coxeter diagram. The group $\Gamma$ is cocompact if and only if the following conditions hold.

- $\mathcal{G}$ contains at least one spherical subdiagram of rank $n$.

- Each spherical subdiagram of rank $n-1$ of $\mathcal{G}$ can be extended in exactly two ways to a spherical subdiagram of rank $n$ of $\mathcal{G}$.

Proposition 1.10 (Finite covolume criterion in CoxIter). Let $\Gamma<$ Isom $\mathcal{H}^{n}$ be a Coxeter group and $\mathcal{G}$ be its Coxeter diagram. The group $\Gamma$ has finite covolume if and only if the following conditions hold.

- $\mathcal{G}$ contains at least one spherical subdiagram of rank $n$ or one parabolic subdiagram of rank $n-1$.

- Each spherical subdiagram of rank $n-1$ of $\mathcal{G}$ can be extended in exactly two ways to one of the following type of subdiagrams of $\mathcal{G}$ :

* a spherical diagram of rank $n$; or

* a parabolic diagram of rank $n-1$.

\subsection{Arithmeticity}

Definition 10. Let $G:=\left(G_{i, j}\right) \in \operatorname{Mat}(n, K)$ be a square matrix with coefficients in a field $K$. A cycle of length $k$, or $k$-cycle, in $G$ is a product $G_{i_{1}, i_{2}} \cdot G_{i_{2}, i_{3}} \cdot \ldots \cdot G_{i_{k-1}, i_{k}} \cdot G_{i_{k}, i_{1}}$. Such a cycle is denoted by $G_{\left(i_{1}, \ldots, i_{k}\right)}$. If the $i_{j}$ are all distinct, the cycle is called irreducible.

Observe that each cycle is a product of irreducible cycles.

Definition 11. Let $\mathcal{G}$ be an undirected graph. A cycle, or closed walk, in $\mathcal{G}$ is a sequence $\left(v_{i_{1}}, \ldots, v_{i_{m}}, v_{i_{1}}\right)$ of adjacent vertices of $\mathcal{G}$. We say that the cycle is simple if all the $v_{i_{j}}$ are different.

Using Theorem 3.1 and the remarks of [24], we get the following result.

Theorem 1.11 [24]. Let $\Gamma$ be a non-cocompact hyperbolic Coxeter group of finite covolume and let $G$ be its Gram matrix. Then $\Gamma$ is arithmetic if and only if all the cycles of the matrix $2 \cdot G$ are rational integers.

Corollary 1.12. Let $\Gamma=(W, S)$ be a non-cocompact hyperbolic Coxeter group of finite covolume and let $G^{\prime}=2 \cdot G$, where $G$ is its Gram matrix. If the Coxeter graph of $\Gamma$ contains no dotted edges, then we have the following result.

$\Gamma$ is arithmetic if and only if the following two conditions are satisfied:

(i) for every $s, t \in S$, we have $m(s, t) \in\{\infty, 2,3,4,6\}$; and

(ii) every irreducible cycle $G_{\left(i_{1}, \ldots, i_{k}\right)}^{\prime}$ of length at least 3 in $G^{\prime}$ lies in $\mathbb{Z}$.

In particular, a necessary condition for $\Gamma$ to be arithmetic is $G \in \operatorname{Mat}(|S|, \mathbb{Q}[\sqrt{2}, \sqrt{3}])$.

From now on, we suppose that $\Gamma=(W, S)$ is a non-cocompact hyperbolic Coxeter group of finite covolume which satisfies condition (i) of Corollary 1.12 and we let $G^{\prime}=2 \cdot G$. If $s \in S$ 

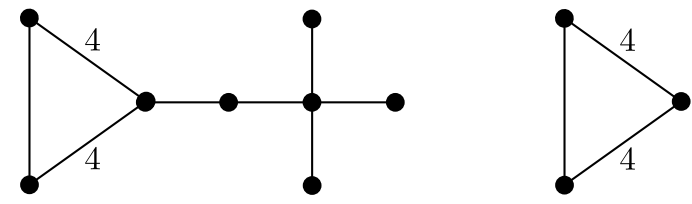

Figure 3. Two equivalent graphs for the arithmeticity criterion.

is a leaf of the Coxeter graph (meaning that $m(s, t)=2$ for every $t \in S \backslash\{s\}$ except for one vertex), then it is clear that $s$ cannot be a member of a non-zero irreducible cycle. Therefore, we can forget this vertex for the test. Applying this idea successively, we see that we can collapse every non-closed path of the graph. For example, the two Coxeter graphs of Figure 3 are equivalent for the arithmeticity criterion.

Now, if $G_{\left(i_{1}, \ldots, i_{k}\right)}^{\prime}$ is an irreducible $k$-cycle, then it corresponds to a simple cycle in the Coxeter graph (see Definition 11) if and only if it is non-zero. Thus, it is sufficient to consider simple cycles in the Coxeter graph. This can be summarized in the next proposition.

Proposition 1.13 (Arithmetic criterion in CoxIter). Let $\Gamma=\left(W, S=\left\{s_{1}, \ldots, s_{d}\right\}\right)$ be a non-cocompact hyperbolic Coxeter group of finite covolume and let $\mathcal{G}$ be its Coxeter graph. We suppose that $\mathcal{G}$ contains no dotted line. Then, $\Gamma$ is arithmetic if and only if the two following conditions are satisfied.

(i) For every $s, t \in S$, we have $m(s, t) \in\{\infty, 2,3,4,6\}$.

(ii) For every simple cycle $\left(s_{1}, \ldots, s_{k}, s_{1}\right)$ in $\mathcal{G}$, the product $2^{k} \cdot \prod_{i=1}^{k-1} \cos \left(\pi / m\left(s_{i}, s_{i+1}\right)\right)$. $\cos \left(\pi / m\left(s_{k}, s_{1}\right)\right)$ is an integer. Moreover, it is sufficient to test this condition in the graph obtained by collapsing every non-closed path.

REMARK 3. If the graph contains dotted lines, we cannot decide the arithmeticity of the group only by looking at the weights $m(s, t)$ in the graph. In order to extend Proposition 1.13, we need to know all the values in the Gram matrix. If there is a dotted line between vertices $i$ and $j$, a necessary condition for arithmeticity is that $4 \cdot G_{i j}^{2} \in \mathbb{Z}$.

\section{Algorithms}

In what follows, we use the notations of the program. In particular, the $r$ vertices of the Coxeter graph are labelled $0, \ldots, r-1$.

\subsection{Euler characteristic and the $f$-vector}

Here, we present the main steps for the computation of the Euler characteristic and the $f$ vector. The main steps are the following.

(1) We find all paths (simple walks with all edges labelled with a 3) starting from every vertex. Note that a single vertex is such a path.

(2) We extend these paths to finite and affine irreducible Coxeter groups.

(3) We compute all the possible products of groups.

(4) We count these products with their orders and multiplicities. The Euler characteristic is then computed with Proposition 1.2 (by taking into account the orders of finite irreducible spherical Coxeter groups in Table 1).

Note that, using (1)-(3), and Theorems 1.4 and 1.5, we can compute the $f$-vector of $\Gamma$.

First, we use a depth-first search algorithm to explore the graph from every vertex and to find any subgraph $A_{m}$ (function CoxIter::DFS). For each such $A_{m}$, we try to extend it to other 
connected spherical and Euclidean graphs (function CoxIter::addGraphsFromPath).

Once the connected graphs are found, we compute all the possible products. Counting them with their multiplicities gives the $f$-vector and the Euler characteristic.

\subsection{Arithmeticity}

To check the arithmeticity of a given Coxeter group, we use Proposition 1.13. The code for the test is in the Arithmeticity class.

First, we check whether the graph contains no dotted edge, if it is non-cocompact and if all the edge labels $m(s, t)$ lie in the set $\{\infty, 2,3,4,6\}$. These verifications are done in the beginning of the Arithmeticity::test function. At this point, we know that the coefficients of the matrix $2 \cdot G$ can take the values $\{0,-1,-2,2,-\sqrt{2},-\sqrt{3}\}$. We use multiple calls to the function Arithmeticity::collapseQueues to collapse all non-closed paths of the graph (regardless of the labels which appear in these paths) to reduce the computation time of the determination of the cycles.

For each vertex $v$, we look for simple cycles passing through $v$ (note that we only look at cycles which contain vertices $j$ with $v \leqslant j$ to avoid multiple counting of the graphs). This is done by using Arithmeticity::findCycles(v,v). For each cycle, we see if the number of edges labelled with 4 and 6 , respectively, along the cycle is even (labels 3 and $\infty$ are not an obstacle to arithmeticity at this point).

As explained in Remark 3, if the graph contains dotted lines, we need to determine all the relevant entries of the Gram matrix in order to decide about the arithmeticity of the group. In this case, CoxIter will indicate what are the conditions for the group to be arithmetic. The program would give conditions in the following way.

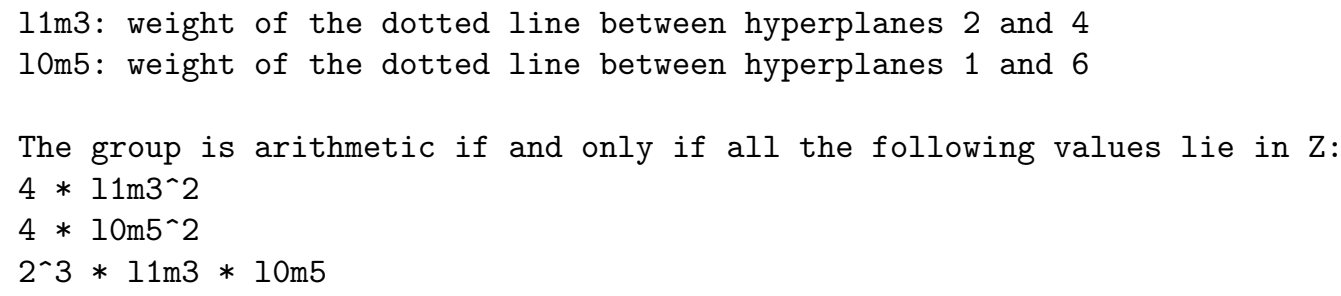

\section{Using CoxIter: some examples}

In this section, we present some worked examples in dimensions 13, 16, 17, 18 and 19 . In dimension 18 we compute the invariants for three groups given by Vinberg in a recent article [25]. The complete documentation of the program can be found online at http: //coxiter.rgug.ch.

\subsection{Two arithmetic groups of Vinberg}

We start with the arithmetic groups $\Sigma_{16}<\operatorname{Isom} \mathcal{H}^{16}$ and $\Sigma_{17}<\operatorname{Isom} \mathcal{H}^{17}$ (see Figure 4 and $[23])$.

The program is called with parameters '- $\mathrm{fv}$-compactness -arithmeticity' (we want to test the cocompactness, the arithmeticity and finite covolume) and the output is given in Table 2. We see that the values match the theoretical results. For the Euler characteristic, see $[\mathbf{1 8}$, Theorem 22], and for a correction factor $2^{k}$, where $k$ is the number of symmetry axis of the graph, see $[\mathbf{1 9}, \S 6]$. In our case, $k=2$. 

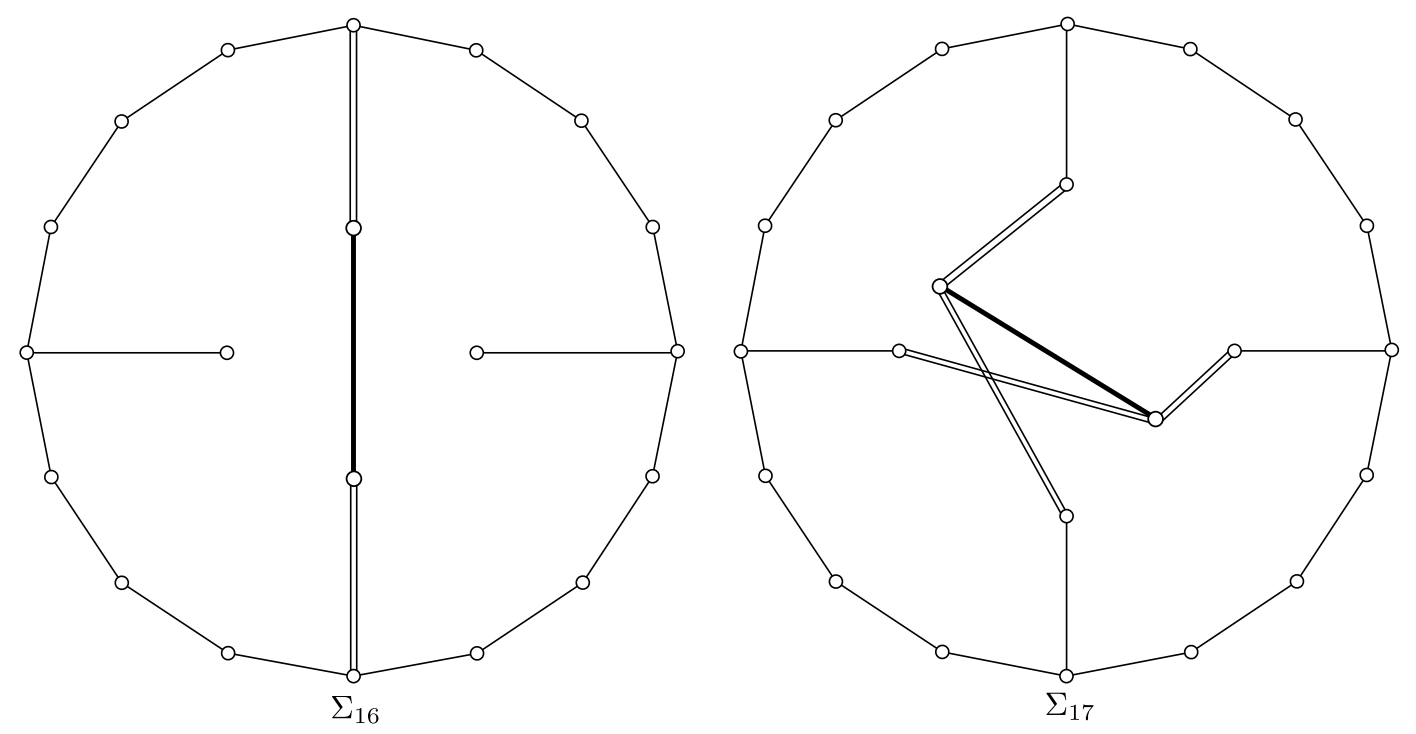

FiguRE $4 . \Sigma_{16}$ and $\Sigma_{17}$.

\subsection{An arithmetic group of McLeod}

In his paper [13], McLeod constructed the maximal reflection groups in the automorphism groups of the quadratic forms $-3 x_{0}+x_{1}^{2}+\ldots+x_{n}^{2}$ for $2 \leqslant n \leqslant 13$, using Vinberg's algorithm. We give here the invariants for $n=13$ (see Figure 5 ). The program is called with parameters '-fv -compactness' (we want to test the cocompactness and finite covolume), and the output is given in Table 3 .

\subsection{A free product with amalgamation in dimension 18}

In a recent paper [25], Vinberg explains how to construct a non-arithmetic group $\Gamma$ in dimension 18 as a free product with amalgamation $\Gamma=\Gamma_{1} \star_{\Gamma_{0}} \Gamma_{2}$, where $\Gamma_{1}$ and $\Gamma_{2}$ are two non-commensurable arithmetic hyperbolic Coxeter groups ( $\Gamma$ is a mixture in the sense of Gromov-Piatetski-Shapiro). Geometrically, we suppose that the two associated polyhedra $P_{1}$ and $P_{2}$ of the groups $\Gamma_{1}, \Gamma_{2}<\operatorname{Isom} \mathcal{H}^{18}$ have an isometric facet $P_{0}$. In this setting, the associated polyhedron $P$ of $\Gamma$ is the gluing of $P_{1}$ and $P_{2}$ along their common facet $P_{0}$. We use

TABLE 2. Output of CoxIter for $\Sigma_{16}$ and $\Sigma_{17}$.

\begin{tabular}{lll}
\hline Invariant & $\Sigma_{16}$ & $\Sigma_{17}$ \\
\hline Cocompact & No & No \\
Finite covolume & Yes & Yes \\
Arithmetic & Yes & Yes \\
f-vector & $(325,2804,11914,33164$, & $(807,7586,33960,98184,206120$, \\
& $67410,105462,130646,130062$, & $332982,427584,444428,377232,262050$, \\
& $104670,68042,35490$, & $148500,68076,24884,7089,1518$, \\
Alt. sum of the & $14658,4690,1122,189,20,1)$ & $230,22,1)$ \\
comp. of the f-vector & 0 & 2 \\
Euler characteristic & 2360171042879569920000 & 0 \\
Running time & $0.6 \mathrm{~s}$ & $2.7 \mathrm{~s}$ \\
\hline
\end{tabular}




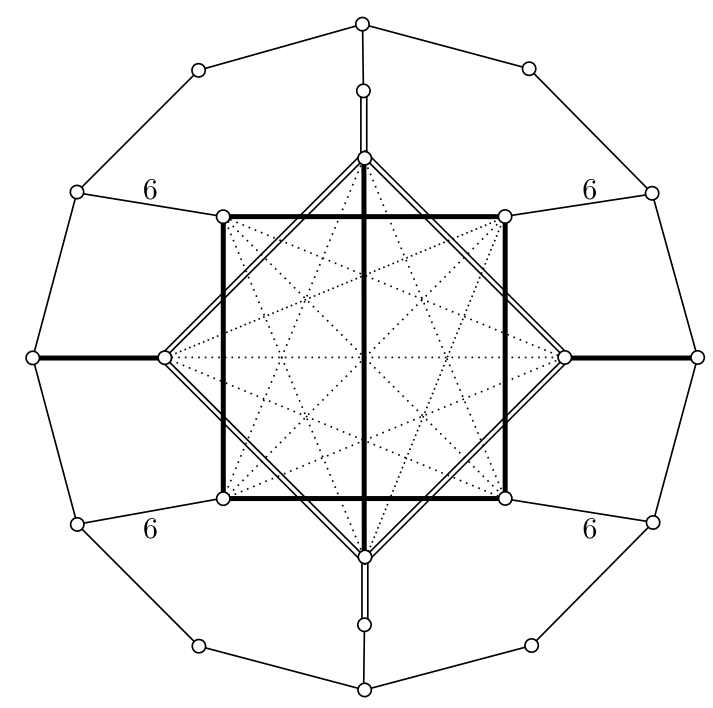

FigURE 5. Group of reflections related to automorphism group of the quadratic form $-3 x_{0}+x_{1}^{2}+\ldots+x_{13}^{2}$.

CoxIter to compute the invariants of the groups $\Gamma_{1}, \Gamma_{2}$ and $\Gamma$ and we check that the covolume of $\Gamma$ is indeed the sum of the covolumes of the two components.

3.3.1. First component of the product. Information about the construction of the first component $\Gamma_{1}$ of the product are given in [10] and [25]. The generators $\left\{s_{1}, \ldots, s_{37}\right\}$ of $\Gamma_{1}$ are given by the roots of a certain integral lattice $L_{1}$ (in fact, $L_{1}$ is the unique odd unimodular quadratic lattice of signature $(18,1)$ ).

We use the terminology and present the results of [10]. There are 22 long roots (that is roots of norm two) and fifteen short roots (that is roots of norm one). The action of the symmetry group of the graph (which is isometric to $S_{4}$; see below) splits the short roots into two orbits of size three and twelve. The three roots are called roots of the first kind while the twelve other elements are called roots of the second kind. The diagram $\Sigma^{*}$ of the long roots is presented in Figure 6(a). If we consider the six 'long edges' (or 'tetrahedral edges') of Figure 6(b), then each pair of opposite edges gives rise to a root of the first kind. Hence, we get the three vertices of the first kind 18, 25, 26 connected, respectively, to vertices $(17,22),(1,9)$ and $(5,13)$ by a double edge. The Figure 6(c) depicts the connection of one vertex of the second kind with the graph $\Sigma^{*}$. We then let the symmetry group of $\Sigma^{*}$ (see below) act on the triple of

TABLE 3. Output of CoxIter for the reflection group corresponding to the automorphism group of the quadratic form $-3 x_{0}+x_{1}^{2}+\ldots+x_{13}^{2}$ (see Figure 5 ).

\begin{tabular}{ll}
\hline Invariant & Value \\
\hline Cocompact & No \\
Finite covolume & Yes \\
f-vector & $(413,2964,10238,22761,36024,42265$, \\
& $37380,25005,12556,4641,1218,213,22,1)$ \\
Alt. sum of the comp. of the f-vector & 2 \\
Euler characteristic & 0 \\
Running time & $0.18 \mathrm{~s}$ \\
\hline
\end{tabular}



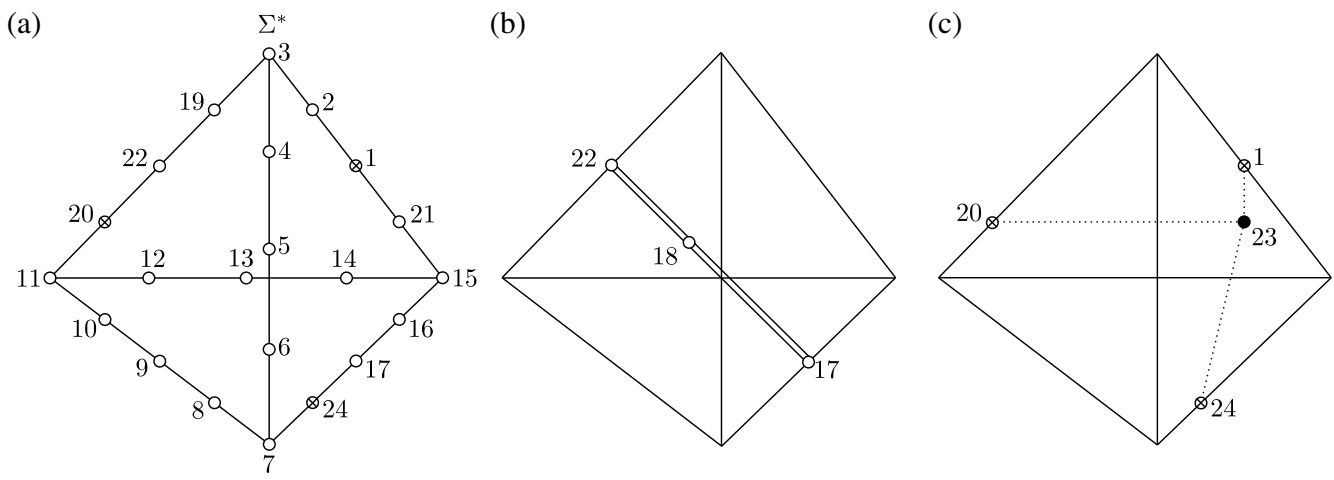

Figure 6. Construction of $\Gamma_{1}<\operatorname{Isom} \mathcal{H}^{18}$ : first component of the product.

vertices $(1,20,24)$ and find 11 other triples, corresponding to the 11 other vertices of the second kind. The 12 triples of vertices are the following: $(1,20,24),(1,6,12),(5,16,20),(5,10,21)$, $(9,16,19),(9,4,14),(13,2,8),(13,19,24),(17,2,10),(17,4,12),(22,8,21),(22,6,14)$. Now, vertices of the first kind are connected among themselves by lines labelled with an $\infty$, while vertices of the second kind are connected among themselves by broken edges. Each vertex of the first kind is connected to each vertex of the second kind either by an $\infty$ or by a broken edge according to the rule explained in $[10, \S 2]$. Finally, we found a Coxeter graph $\Sigma\left(\Gamma_{1}\right)$ with 37 vertices and 171 edges. When given to CoxIter, it produces the output given in Table 4 .

Lemma $3.1[\mathbf{1 0}]$. The symmetry group of the graph $\Sigma\left(\Gamma_{1}\right)$ is isometric to $S_{4}$.

Proof. To determine the symmetry group of the graph of the group $\Gamma_{1}$, we first compute the group of symmetries Sym $\Sigma^{*}$ of the graph $\Sigma^{*}$, presented in Figure 6. It is easy to see that $\operatorname{Sym} \Sigma^{*}$ has order 24 . We consider the automorphisms

$$
\begin{aligned}
& \sigma_{1}=\left(\begin{array}{ll}
22 & 9
\end{array}\right)\left(\begin{array}{ll}
1 & 17
\end{array}\right) \\
& \sigma_{2}=\left(\begin{array}{lll}
1 & 5
\end{array}\right)\left(\begin{array}{ll}
9 & 13
\end{array}\right) \\
& \sigma_{3}=\left(\begin{array}{lll}
22 & 1
\end{array}\right)\left(\begin{array}{ll}
9 & 17
\end{array}\right)
\end{aligned}
$$

and we see that $\sigma_{1} \circ \sigma_{2}$ has order three, $\sigma_{2} \circ \sigma_{3}$ has order three and $\sigma_{1} \circ \sigma_{3}$ has order two. Therefore, $\operatorname{Sym} \Sigma^{*}=S_{4}$. Finally, as explained in [10], we have $\operatorname{Sym} \Sigma^{*} \cong \operatorname{Sym} \Sigma$.

TABLE 4. Output of CoxIter for $\Gamma_{1}$.

\begin{tabular}{ll}
\hline Invariant & Value \\
\hline Cocompact & No \\
Finite covolume & Yes \\
f-vector & $(3839,37842,177812,540624,1197240,2050008,2807602$, \\
& $3135528,2883540,2189924,1369854,700352,288801$, \\
Alt. sum of the comp. of the f-vector & $94113,23497,4282,525,37,1)$ \\
Euler characteristic & $-\frac{109638854849}{22028263066875985920000}$ \\
Volume & $\pi^{9} \cdot \frac{10963854849}{1482580623111880900608000000}$ \\
Running time & $55 \mathrm{~s}$ \\
\hline
\end{tabular}




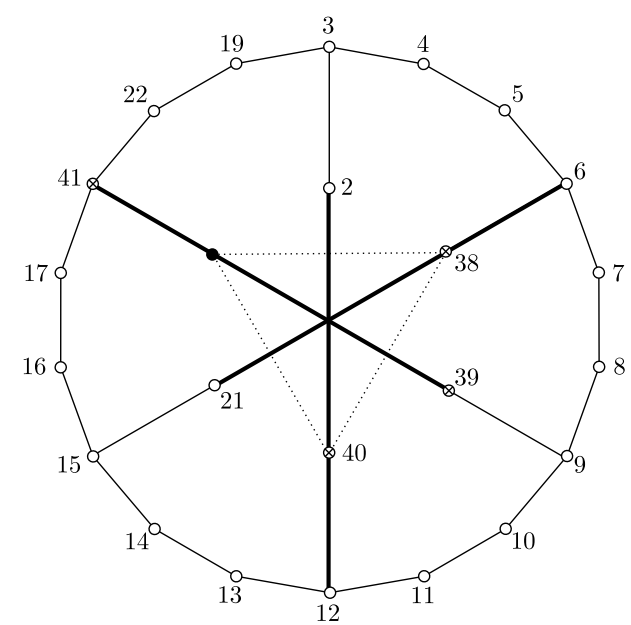

FiguRE 7. $\Gamma_{2}<\operatorname{Isom} \mathcal{H}^{18}$ : second component of the product.

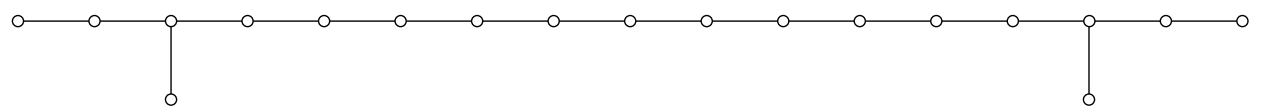

FiguRE 8. $\Gamma_{0}<\operatorname{Isom} \mathcal{H}^{17}$.

3.3.2. Second component of the product. The lattice for the second group is $L_{2}=L_{0} \oplus$ $\mathbb{Z} e \subset \mathbb{R}^{18,1}$, where:

- $L_{0}$ is the unique even unimodular quadratic lattice of signature $(17,1)$ (its maximal subgroup generated by reflections is $\Gamma_{0}$, the hyperbolic Coxeter group whose associated fundamental polyhedron is $P_{0}$, the common facet of $P_{1}$ and $P_{2}$ ); and

$-e$ is a long root of norm two.

The graph of the group $\Gamma_{2}<\operatorname{Isom} \mathcal{H}^{18}$ is presented in Figure 7 and its computed invariants in Table 5.

3.3.3. Amalgamated product. As written previously, we want to construct the amalgamated product of the groups $\Gamma_{1}$ and $\Gamma_{2}$. We see that they have a common hyperbolic subgroup $\Gamma_{0}$ of signature $(17,1,1)$ (see Figure 8). The hyperplane in $\mathcal{H}^{18}$, which contains the associated polytope $P_{0}$, is the one corresponding to the black dot in the graphs of $\Gamma_{1}$ and $\Gamma_{2}$.

TABle 5. Output of CoxIter for $\Gamma_{2}$.

\begin{tabular}{ll}
\hline Invariant & Value \\
\hline Cocompact & No \\
Finite covolume & Yes \\
f-vector & $(535,5160,24876,79590,188352,348012,517247,628599$, \\
& $629544,520631,354651,197676,89148,31977,8892,1843$, \\
Alt. sum of the comp. of the f-vector & 0 \\
Euler characteristic & $-\frac{109638854849}{22600997906614761553920000}$ \\
Volume & $\pi^{9} \cdot \frac{109638854849}{1521127719312789804023808000000}$ \\
Running time & $3.2 \mathrm{~s}$ \\
\hline
\end{tabular}


A presentation for the product $\Gamma=\Gamma_{1} \star_{\Gamma_{0}} \Gamma_{2}$ is obtained as follows.

- Start with the presentation of $\Gamma_{1}=\left\langle s_{1}, \ldots, s_{37} \mid\left(s_{i} \cdot s_{j}\right)^{m_{i j}}\right\rangle$.

- Remove the vertex 23, corresponding to the hyperplanes containing $P_{0}$.

- Add four generators $s_{38}, s_{39}, s_{40}, s_{41}$ corresponding to the crossed vertices of $\Gamma_{2}$.

- The relations between these four new generators and the generators $s_{2}, \ldots, s_{17}, s_{19}, s_{21}$, $s_{22}$ are according to Figure 7 .

- There is no relation between any of the generators $s_{38}, s_{39}, s_{40}, s_{41}$ and any of $s_{1}$, $s_{18}, s_{20}, s_{24}, \ldots, s_{37}$, meaning that the corresponding $m_{i j}$ are infinity. Note that at this point we do not know if the corresponding hyperplanes are parallel or ultraparallel (or, equivalently, if in the graph we have dotted or bold edges). This means that we cannot fully determine the $f$-vector (that is the number $f_{0}$ of vertices is unknown). However, this does not influence the computation for the Euler characteristic.

The output of CoxIter for the product $\Gamma$ is given in Table 6 . As expected, we find the equality $\chi(\Gamma)=\chi\left(\Gamma_{1}\right)+\chi\left(\Gamma_{2}\right)$. Moreover, using Proposition 1.6, we find that the number $f_{0}$ of vertices of the polyhedron is 4212 .

\subsection{An arithmetic group in dimension 19}

Kaplinskaja and Vinberg described in [10] the construction of an arithmetic hyperbolic Coxeter group $\Gamma_{19}$ in Isom $\mathcal{H}^{19}$ related to the standard quadratic form $-x_{0}^{2}+x_{1}^{2}+\ldots+x_{19}^{2}$. This construction is similar to the first component of the free amalgamated product in $\S 3.3 .1$.

In this section, we use the terminology of [10]. There are 25 long roots (depicted in the diagram $\Sigma^{*}$ of Figure 9), five roots of the first kind (such as the vertex 19 in Figure 9(b)) and twenty roots of the second kind (such as the vertex 24 in Figure 9(c)). In order to explain how to connect the vertices of the first and second kind to the graph $\Sigma^{*}$, we first compute the automorphism group of the graph $\Sigma^{*}$.

Lemma 3.2 [10]. The automorphism group of $\Sigma^{*}$ is isomorphic to $S_{5}$.

Proof. Using the same method as in Lemma 3.1, we find the four generators of Sym $\Sigma^{*}$ in order to conclude that

$$
\begin{aligned}
& \sigma_{1}=(274)(14 \text { 20) (16 18) (2 12) (21 22) (26 10) } \\
& \sigma_{2}=\left(\begin{array}{l}
4 \\
6
\end{array}\right)(820)(2 \text { 25) (10 21) (16 22) (18 26) } \\
& \sigma_{3}=\left(\begin{array}{l}
6 \\
25
\end{array}\right)\left(\begin{array}{l}
4 \\
16
\end{array}\right)(14 \text { 20) (2 22) (12 21) (18 27) } \\
& \sigma_{4}=(220)(1826)(825) \text { (21 22) (12 14) (10 16). }
\end{aligned}
$$

3.4.1. Vertices of the first and second kind. In order to determine the vertices of the first kind and their connections to the graph $\Sigma^{*}$, we consider the triple of vertices $(27,26,18)$

TABle 6. Output of CoxIter for $\Gamma$.

\begin{tabular}{ll}
\hline Invariant & Value \\
\hline f-vector & $(4212,41464,195047,594510,1321044,2271012,3123717,3503919,3235974$, \\
& $2467015,1548147,793376,327561,106710,26575,4814,583,40,1)$ \\
Euler characteristic & $-\frac{8661469533071}{1738538300508827811840000}$ \\
Volume & $\pi^{9} \cdot \frac{8661469533071}{117009824562522292617216000000}$ \\
Running time & $74 \mathrm{~s}$ \\
\hline
\end{tabular}


(a)

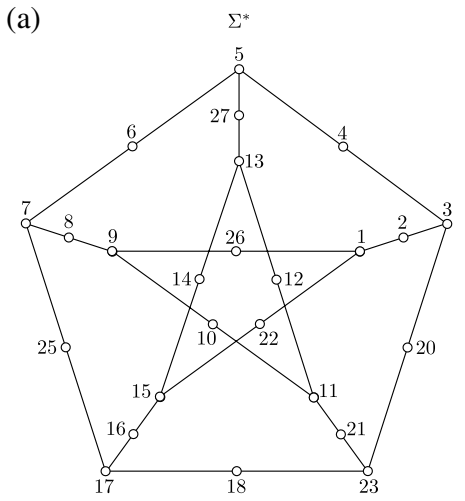

(b)

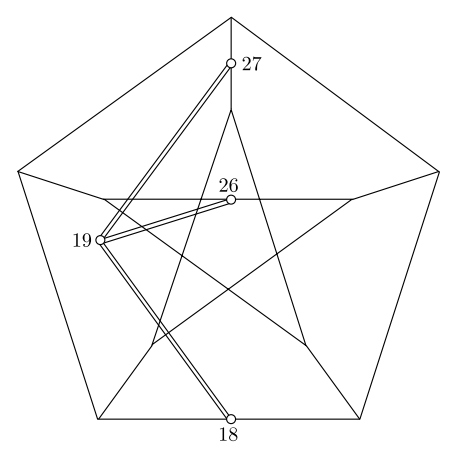

(c)

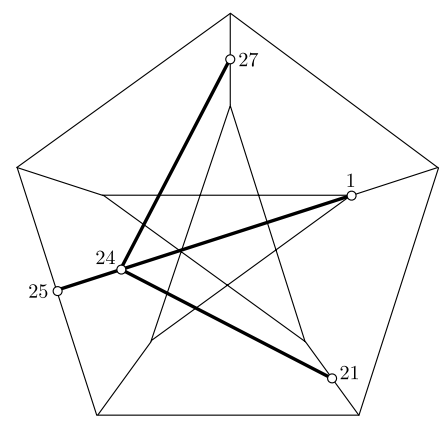

Figure 9. Construction of $\Sigma_{19}<\operatorname{Isom} \mathcal{H}^{19}$.

(see Figure 9(b)) and look at its images under action of $\operatorname{Sym} \Sigma^{*} \cong S_{5}$. We find five triples which correspond to five vertices of the first kind. These are

$$
(27,26,18), \quad(2,12,25), \quad(21,22,6), \quad(16,10,4), \quad(8,14,20) .
$$

The vertices of the first kind are connected among themselves by bold edges.

To determine the vertices of the second kind and their connections to the graph $\Sigma^{*}$, we consider the quadruple of vertices $(27,1,21,25)$ (see Figure $9(\mathrm{c}))$ and look at its images under the action of Sym $\Sigma^{*}$. We find twenty quadruples which correspond to the twenty vertices of the second kind. These are

$$
\begin{array}{lllll}
(1,21,25,27), & (8,22,23,27), & (4,8,15,21), & (4,11,22,25), & (2,10,17,27) \\
(9,16,20,27), & (4,9,14,18), & (4,12,17,26), & (2,8,13,18), & (13,20,25,26) \\
(3,10,14,25), & (3,8,12,16), & (1,6,12,18), & (6,14,23,26), & (2,6,11,16) \\
(6,10,15,20), & (2,7,14,21), & (7,12,20,22), & (5,16,21,26), & (5,10,18,22) .
\end{array}
$$

The vertices of the second kind are connected among themselves by broken edges.

A vertex of the first kind and a vertex of the second kind are connected by a double edge or a broken edge according to the rule described in [10, $\S 2$, p. 196].

3.4.2. Summary and computations. Finally, the connections between the 50 vertices of the group $\Gamma_{19}$ are as follows:

- 30 simple edges in $\Sigma^{*}$;

- a complete graph with 5 vertices (vertices of the first kind, bold edges);

- 15 double edges (vertices of the first kind and $\Sigma^{*}$ );

- a complete graph with 20 vertices (vertices of the second kind, broken edges);

- 80 bold edges (vertices of the second kind and $\Sigma^{*}$ ); and

- 100 edges between vertices of the first and second kind.

The output of CoxIter for the group $\Gamma_{19}$ is presented in Table 7 . 
TABLE 7. Output of CoxIter for $\Gamma_{19}$.

\begin{tabular}{ll}
\hline Invariant & Value \\
\hline Cocompact & No \\
Finite covolume & Yes \\
f-vector & $(27841,292340,1429615,4465955,10081519,17518035$, \\
& $24310230,27542850,25791030,20062168,12956240,6908365$, \\
& $3009960,1054645,290315,60660,9125,905,50,1)$ \\
Alt. sum of the comp. & \\
of the f-vector & 2 \\
\hline
\end{tabular}

Appendix A. Example of the introduction

The output presented in the introduction is the group $\Gamma$ generated by reflections in the group of units of the Lorentzian quadratic form $-x_{0}^{2}+x_{1}^{2}+\ldots+x_{14}^{2}$ of signature $(14,1)$ described in $[\mathbf{2 1}]$. We describe the group $\Gamma$ by means of the text file '14-vinb85.coxiter', as shown in Figure A.1.

Remarks.

- The first line indicates that the Coxeter group $\Gamma$ has 17 generators and that it is a subgroup of Isom $\mathcal{H}^{14}$

- Each of the remaining 17 lines describes one edge of the Coxeter diagram: the first two numbers are the labels of the generators and the third one is the label of the edge ( $\mathrm{a}$ ' 0 ' indicates a bold edge).

\section{Appendix B. Program testing and some values}

To test the accuracy of the program, we ran it on a collection of (around 800) groups for which some of the invariants were known. These graphs can be found in the graphs/folder of the source code. Except for a few graphs, the name of each file goes

$$
\text { dimension - reference_page number - name or number of the graph. }
$$

The reference is as given in Table C.1.

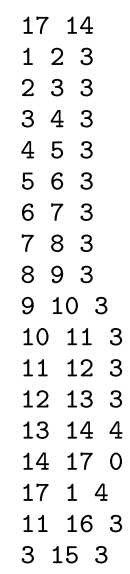

Content of the text file

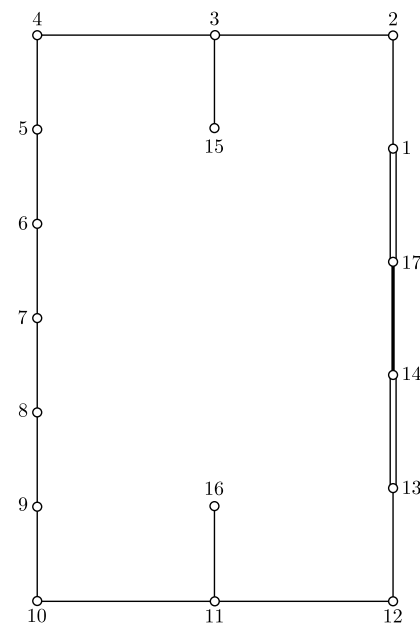

Corresponding Coxeter diagram

Figure A.1. 14-vinb85.coxiter 


\section{B.1. Euler characteristic}

The groups of Table C.2 were tested.

\section{B.2. $f$-vector}

The $f$-vectors of the groups of Table C.3 were tested. The alternating sum of the components of the $f$-vectors of the groups $[\mathbf{8}, \mathbf{2 0}],[\mathbf{1 4}$, Appendix C] and [23] were tested (see Proposition 1.6).

\section{B.3. Cocompactness and finite covolume criterion}

The groups of Table C.4 were tested for cocompactness and the ones of Table C.5 were tested for the finite covolume.

\section{B.4. Arithmeticity}

The groups of Table C.6 were tested.

\section{B.5. Some more complicated Coxeter graphs}

Using cocompact groups in dimensions five and six we constructed twelve sequences $\mathcal{S}_{i}=$ $\left\{\Gamma_{0}<\Gamma_{1}<\ldots<\Gamma_{9}\right\}$ of ten hyperbolic Coxeter groups such that $\Gamma_{i}$ is of index two in $\Gamma_{i+1}$. These groups allowed us to test CoxIter on more complicated graphs (the number of nodes ranges from 7 to 775$)$.

\section{Appendix C. Tables}

TABLE C.1. Correspondence between bibliography and file names.

\begin{tabular}{ll}
\hline Reference in the paper & Name of the file \\
\hline$[\mathbf{5}]$ & ess96 \\
{$[\mathbf{6}]$} & $\mathrm{ftz0} 7$ \\
{$[\mathbf{8}]$} & $\mathrm{jkrt}$ \\
{$[\mathbf{1 3}]$} & $\mathrm{mcl11}$ \\
{$[\mathbf{1 4}]$} & per09 \\
{$[\mathbf{2 0}]$} & tum04 \\
\hline
\end{tabular}

TABLE C.2. Euler characteristic of some groups.

\begin{tabular}{lll}
\hline Reference & Group & Theoretical value \\
\hline$[\mathbf{8}, \mathbf{2 0}, \mathbf{2 3}]$ & & \\
{$[\mathbf{1 4}$, Appendix C] } & All groups of odd dimension & Remark 2 \\
{$[\mathbf{6}]$} & 13 compact simplicial prisms in $\mathcal{H}^{4}$, page 117 & {$[\mathbf{6}]$} \\
{$[\mathbf{8}]$} & 14 groups in $\mathcal{H}^{4}$ & {$[\mathbf{8}]$} \\
{$[\mathbf{8}]$} & 3 groups in $\mathcal{H}^{6}$ & {$[\mathbf{8}]$} \\
{$[\mathbf{1 4}$, Appendix C] } & Bugaenko $P_{6} \subset \mathcal{H}^{6}$, Bugaenko $P_{8} \subset \mathcal{H}^{8}$ & {$[\mathbf{1 1}]$} \\
{$[\mathbf{8}]$} & 4 groups in $\mathcal{H}^{8}$ & {$[\mathbf{8}]$} \\
{$[\mathbf{2 3}]$} & $\Sigma_{10}$ and a subgroup & {$[\mathbf{1 7}$, Theorem 22] } \\
{$[\mathbf{7}]$} & $P_{1}^{10}$ & {$[\mathbf{7}]$} \\
{$[\mathbf{2 3}]$} & $\Sigma_{12}$ and a subgroup & {$[\mathbf{1 7}$, Theorem 22] } \\
{$[\mathbf{2 3}]$} & $\Sigma_{14}$ & {$[\mathbf{1 7}$, Theorem 22] } \\
{$[\mathbf{2 3}]$} & $\Sigma_{16}$ & {$[\mathbf{1 7}$, Theorem 22] } \\
\hline
\end{tabular}


TABLE C.3. $f$-vector of some groups.

\begin{tabular}{ll}
\hline Group & Theoretical value \\
\hline All groups of $[\mathbf{8}]$ & Simplexes \\
All pyramids in $\mathcal{H}^{3}$ of $[\mathbf{2 0}]$ & \\
Bugaenko $P_{6}$ & {$[\mathbf{1 1}]$} \\
Bugaenko $P_{8}$ & {$[\mathbf{2 6}]$} \\
Birectified 5-simplex & http://en.wikipedia.org/wiki/Rectified_5-simplexes \\
\hline
\end{tabular}

TABLE C.4. Cocompactness.

\begin{tabular}{lll}
\hline Reference & Cocompact & Groups \\
\hline$[\mathbf{5}]$ & Yes & Groups in Isom $\mathcal{H}^{4}$ \\
{$[\mathbf{1 4}$, Appendix C] } & Yes & All groups \\
{$[\mathbf{6}]$} & Yes & 13 compact simplicial prisms in $\mathcal{H}^{4}$, page 117 \\
{$[\mathbf{9}]$} & Yes & $\overline{B H}_{3}, \bar{J}_{3}, \overline{D H}_{3}, \widehat{A B}_{3}, \bar{K}_{3}, \widehat{A H}_{3} \widehat{B B}_{3}$ \\
& & $\widehat{B H}_{3}, \overline{H H}_{3}, \bar{H}_{4}, \overline{B H}_{4}, \overline{D H}_{4}, \bar{K}_{4}, \overline{A F}_{4}$ \\
\hline$[\mathbf{2 0}]$ & No & All groups \\
{$[\mathbf{2 0}]$} & No & All groups obtained by removing a polar \\
{$[\mathbf{2 3}]$} & No & $\left\{\Sigma_{n}: 10 \leqslant n \leqslant 17\right\}$ \\
{$[\mathbf{9}]$} & No & $\bar{V}_{3}, \bar{R}_{3}, \bar{P}_{3}, \overline{B V}_{3}, \bar{O}_{3}, \bar{Y}_{3}, \overline{H V}_{3}, \overline{B P}_{3}, \overline{D V}_{3}, \bar{N}_{3}, \bar{Z}_{3}$ \\
& & $\overline{B R}_{3}, \overline{H P}_{3}, \widehat{A V}_{3}, \overline{D P}_{3}, \bar{M}_{3}, \overline{V P}_{3}, \widehat{B V}_{3}, \widehat{C R}_{3}, \overline{H V}_{3}$ \\
& & $\widehat{V V}_{3}, \widehat{R R}_{3}, \widehat{P P}_{3}, \bar{S}_{4}, \bar{R}_{4}, \bar{P}_{4}, \bar{O}_{4}, \bar{N}_{4}, \bar{M}_{4}, \overline{B P}_{4}, \overline{F R}_{4}$ \\
& & $\overline{D P}_{4}, \bar{U}_{5}, \bar{S}_{5}, \bar{X}_{5}, \bar{Q}_{5}, \bar{R}_{5}, \bar{P}_{5}, \bar{O}_{5}, \bar{N}_{5}, \overline{A U}_{5}, \bar{M}_{5}$ \\
& & $\bar{L}_{5}, \widehat{U R}_{5}, \bar{S}_{6}, \bar{Q}_{6}, \bar{P}_{6}, \bar{T}_{7}, \bar{S}_{7}, \bar{Q}_{7}, \bar{P}_{7}, \bar{T}_{8}, \bar{S}_{8}, \bar{Q}_{8}, \bar{P}_{8}$ \\
\hline
\end{tabular}

TABLE C.5. Finite covolume.

\begin{tabular}{lll}
\hline Reference & Finite covolume & Groups \\
\hline$[\mathbf{6}]$ & Yes & 13 compact simplicial prisms in $\mathcal{H}^{4}$, page 117 \\
{$[\mathbf{8}]$} & Yes & All groups \\
{$[\mathbf{1 4}$, Appendix C] } & Yes & All groups \\
{$[\mathbf{2 0}]$} & Yes & All groups \\
{$[\mathbf{2 3}]$} & Yes & $\left\{\Sigma_{n}: 10 \leqslant n \leqslant 17\right\}$ \\
\hline$[\mathbf{2 0}]$ & No & Every polar was removed to create 387 \\
& & groups which do not have finite covolume \\
\hline
\end{tabular}

TABle C.6. Arithmeticity.

\begin{tabular}{lll}
\hline Reference & Arithmetic & Groups \\
\hline$[\mathbf{8}]$ & Yes (see $[\mathbf{9}])$ & $\bar{S}_{6}, \bar{Q}_{6}, \bar{P}_{6}, \bar{T}_{7}, \bar{S}_{7}, \bar{Q}_{7}, \bar{P}_{7}, \bar{T}_{8}, \bar{S}_{8}, \bar{Q}_{8}, \bar{P}_{8}$ \\
{$[\mathbf{2 3}]$} & Yes & $\left\{\Sigma_{n}: 10 \leqslant n \leqslant 17\right\}$ \\
{$[\mathbf{2 0}]$} & & All groups \\
\hline
\end{tabular}

Acknowledgements. The author wishes to thank his advisor Prof. Ruth Kellerhals for numerous discussions, helpful comments and advice. The author would also like to thank Matthieu Jacquemet for his comments and the idea of the name CoxIter and Scott Thomson for the early testing of the program and his comments. 


\section{References}

1. R. Borcherds, 'Automorphism groups of Lorentzian lattices', J. Algebra 111 (1987) 133-153.

2. N. Bourbaki, Groupes et Algebres de Lie (Hermann, Paris, 1968).

3. V. O. Bugaenko, 'Arithmetic crystallographic groups generated by reflections, and reflective hyperbolic lattices', Lie groups, their discrete subgroups, and invariant theory, Advances in Soviet Mathematics 8 (1992) 33-55.

4. H. S. M. Coxeter, 'The complete enumeration of finite groups of the form $R_{i}^{2}=\left(R_{i} R_{j}\right)^{k_{i j}}=1$ ', J. Lond. Math. Soc. 1 (1935) 21-25.

5. F. Esselmann, 'The classification of compact hyperbolic Coxeter $d$-polytopes with $d+2$ facets', Comment. Math. Helv. 71 (1996) 229-242.

6. A. Felikson, P. V. Tumarkin and T. Zehrt, 'On hyperbolic Coxeter $n$-polytopes with $n+2$ facets', Adv. Geom. 7 (2007) 177-189.

7. T. HiLd, 'Cusped hyperbolic orbifolds of minimal volume in dimensions less than 11', PhD Thesis, University of Fribourg, 2007.

8. N. Johnson, R. Kellerhals, J. G. Ratcliffe and S. T. Tschantz, 'The size of a hyperbolic Coxeter simplex', Transform. Groups 4 (1999) 329-353.

9. N. Johnson, R. Kellerhals, J. G. Ratcliffe and S. T. Tschantz, 'Commensurability classes of hyperbolic Coxeter groups', Linear Algebra Appl. 345 (2002) 119-147.

10. I. M. Kaplinskaja and E. B. VinBerg, 'On the groups $O_{18,1}(\mathbb{Z})$ and $O_{19,1}(\mathbb{Z})$ ', Dokl. Akad. Nauk $S S S$ 238 (1978) 1273-1275.

11. R. Kellerhals, 'Hyperbolic orbifolds of minimal volume', Comput. Methods Funct. Theory 14 (2014) 465-481.

12. R. Kellerhals and G. Perren, 'On the growth of cocompact hyperbolic Coxeter groups', European J. Combin. 32 (2011) 1299-1316.

13. J. MCLEOD, 'Hyperbolic reflection groups associated to the quadratic forms $-3 x_{0}^{2}+x_{1}^{2}+\ldots+x_{n}^{2}$, Geom. Dedicata 152 (2011) 1-16.

14. G. Perren, 'Growth of cocompact hyperbolic Coxeter groups and their rate', PhD Thesis, University of Fribourg, 2009, http://homeweb1.unifr.ch/kellerha/pub/DissGPerren09-final.pdf.

15. H. Poincaré, 'Sur la généralisation d'un théorème dEuler relatif aux polyèdres', C. R. Séances Acad. Sci. 117 (1893) 144-145.

16. M. N. Prokhorov, 'The absence of discrete reflection groups with noncompact fundamental polyhedron of finite volume in Lobachevsky space of large dimension', Izv. Math. 2 (1987) 401-411.

17. J. Ratcliffe, Foundations of hyperbolic manifolds, Graduate Texts in Mathematics 149 (Springer, New York, 2006).

18. J. RAtCliffe and S. T. Tschantz, 'Volumes of integral congruence hyperbolic manifolds', J. reine angew. Math. 488 (1997) 55-78.

19. J. Ratcliffe and S. T. Tschantz, 'On volumes of hyperbolic Coxeter polytopes and quadratic forms', J. Geom. Dedicata 163 (2013) 285-299.

20. P. V. Tumarkin, 'Hyperbolic Coxeter $n$-polytopes with $n+2$ facets', Math. Notes 75 (2004) 848-854.

21. E. B. Vinberg, 'On groups of unit elements of certain quadratic forms', Sb. Math. 16 (1972) 17-35.

22. E. B. Vinberg, 'Absence of crystallographic groups of reflections in Lobachevsky spaces of large dimension', Funct. Anal. Appl. 15 (1981) 128-130.

23. E. B. Vinberg, 'Hyperbolic reflection groups', Russian Math. Surveys 40 (1985) 31-75.

24. E. B. Vinberg, Geometry II: spaces of constant curvature, Encyclopaedia of Mathematical Sciences 29 (Springer, Berlin, 1993).

25. E. B. VinBerG, 'Non-arithmetic hyperbolic reflection groups in higher dimensions', Mosc. Math. J. 15 (2015) 593-602.

26. T. Zehrt, 'The covolume of discrete subgroups of Isom $\mathcal{H}^{2 m}$, Discrete Math. 309 (2009) 2284-2291.

\section{R. Guglielmetti}

Université de Fribourg

Chemin du Musée 23

CH-1700 Fribourg

Switzerland

rafael.guglielmetti@unifr.ch 